\title{
Fenología de Tayloria dubyi (Splachnaceae) en las turberas de la Reserva de Biosfera Cabo de Hornos
}

\author{
Phenology of Tayloria dubyi (Splachnaceae) in the peatlands of the Cape Horn Biosphere \\ Reserve
}

\begin{abstract}
JOCELYN JOFRE $1,2,{ }^{*}$, FRANCISCA MASSARDO ${ }^{1,2}$, RICARDO ROZZI ${ }^{1,2,3}$, BERNARD GOFFINET ${ }^{4}$, PAUL MARINO $^{5}$, ROBERT RAGUSO $^{6}$ \& NELSO P. NAVARRO ${ }^{1,7}$
\end{abstract}

\author{
${ }^{1}$ Programa de Magíster en Ciencias, Facultad de Ciencias, Universidad de Magallanes, Casilla 113-D, Avenida Bulnes \\ 01855, Punta Arenas, Chile \\ ${ }^{2}$ Instituto de Ecología y Biodiversidad, Facultad de Ciencias, Universidad de Chile, Casilla 653, Santiago, Chile \\ ${ }^{3}$ Department of Philosophy, University of North Texas, Denton, TX 76201 \\ ${ }^{4}$ Department of Ecology and Evolutionary Biology, 75 N. Eagleville Road, University of Connecticut, Storrs, CT 06269- \\ 3043, USA \\ ${ }^{5}$ Department of Biology, Memorial University, St. John's, NL A1B 3X9, Canada \\ ${ }^{6}$ Department of Neurobiology and Behavior, Seeley G. Mudd Hall, Cornell University, Ithaca, NY 14853-2702, USA \\ ${ }^{7}$ Departamento de Botánica, Instituto de Biociencias, Universidad de São Paulo, C. Postal 11461, São Paulo, Brasil \\ * Autor correspondiente: jocelyn.jofre@umag.cl
}

\begin{abstract}
RESUMEN
Los bosques de la ecorregión subantártica de Magallanes incluyen una alta diversidad de briofitas, superando la diversidad de las plantas vasculares. A pesar de esto, estudios biológicos referentes a fenología de briofitas son inexistentes para esta ecorregión y Chile. A partir del estudio de la fase esporofítica de Tayloria dubyi, musgo endémico de la ecorregión subantártica de Magallanes, proponemos una metodología para estudios fenológicos en briofitas australes. Se definieron cinco fenofases fácilmente distinguibles con lupa de mano, que se registraron mensualmente durante los años 2007 y 2008 en poblaciones de $T$. dubyi en el Parque Etnobotánico Omora y bahía Mejillones en la isla Navarino $\left(55^{\circ} \mathrm{S}\right)$, Reserva de Biosfera Cabo de Hornos. La fase esporofítica (o reproductiva) de T. dubyi presentó un claro patrón estacional. Los esporofitos crecen en noviembre, durante los tres meses siguientes (diciembre-febrero) de la estación reproductiva austral maduran y liberan sus esporas y en marzo ya se encuentran senescentes. Tayloria dubyi pertenece a la familia Splachanceae, musgos en los que se ha detectado entomocoría (dispersión de sus esporas por insectos, específicamente por dípteros) en el Hemisferio Norte. El período de liberación de esporas desde los esporofitos de T. dubyi a comienzos del verano austral, coincide con los meses de mayor actividad de dípteros potencialmente dispersantes de esporas; por lo tanto, la entomocoría también podría ocurrir en la ecorregión subantártica de Magallanes. En suma, nuestro trabajo: (i) define una metodología para estudios fenológicos en briófitas australes, (ii) determina una marcada estacionalidad para la fase esporofítica de $T$. $d u b y i$, y (iii) propone evaluar en investigaciones futuras la ocurrencia de entomocoría en especies de Splachnaceae que habitan en turberas y ecosistemas forestales subantárticos del Hemisferio Sur.
\end{abstract}

Palabras clave: briofitas, ecorregión subantártica de Magallanes, fenología, Reserva de Biosfera Cabo de Hornos, Splachnaceae.

\section{ABSTRACT}

The sub-Antarctic Magellanic ecoregion harbors a high diversity of bryophytes, greater than the species richness of vascular plants. Despite this fact, phenological studies on bryophytes are lacking for this ecoregion and Chile. Based on the study of the sporophytic phase of Tayloria dubyi, an endemic moss from the sub-Antarctic Magellanic ecoregion, we propose a methodology for phonological studies on austral bryophytes. We defined five phenophases, easily distinguishable with a hand-lens, which were monthly recorded during 2007 and 2008 in populations of T. dubyi at the Omora Ethnobotanical Park and Mejillones Bay on Navarino Island $\left(55^{\circ} \mathrm{S}\right)$ in the Cape Horn Biosphere Reserve. The sporophytic (or reproductive) phase of $T$. dubyi presented a clear seasonality. After growing in November, in three months (DecemberFebruary) of the austral reproductive season the sporophytes mature and release their spores; by March they are already senescent. T. $d u b y i$ belongs to the Splachnaceae family for which entomochory (dispersal of spores by insects, specifically Diptera) has been detected in the Northern Hemisphere. The period of spores 
release in T. dubyi coincides with the months of highest activity of Diptera which are potential dispersers of spores; hence, entomochory could also take place in sub-Antarctic Magellanic ecoregion. In sum, our work: (i) defines a methodology for phenological studies in austral bryophytes, (ii) it records a marked seasonality ion the sporophyte phase of $T$. dubyi, and (iii) it proposes to evaluate in future research the occurrence of entomochory in Splachnaceae species growing in the sub-Antarctic peatlands and forest ecosystems in the Southern Hemisphere.

Key words: bryophytes, Cape Horn Biosphere Reserve, phenology reproduction, Splachnaceae, sub-Antartic Magellanic ecoregion.

\section{INTRODUCCIÓN}

Los bosques de la ecorregión subantártica de Magallanes albergan una alta diversidad de especies de briofitas, las que superan en riqueza de especies a las plantas vasculares (Rozzi et al. 2008). Por esta razón, la ecorregión subantártica de Magallanes ha sido propuesta como un "hotspot de diversidad de briofitas" a nivel mundial, donde es posible encontrar más de 750 especies de hepáticas y musgos que, en conjunto, conforman más del $5 \%$ de las especies conocidas en todo el planeta (Rozzi et al. 2004, 2008). A pesar de la alta riqueza y diversidad de briofitas, estudios de fenología de briofitas son inexistentes para esta ecorregión y muy escasos en Chile y el mundo (Jofre 2009). Para el grupo de plantas no vasculares o briofitas (musgos, hepáticas y antocerotes), Stark (2002) ha definido la fenología como "el estudio del crecimiento y los eventos reproductivos en el tiempo". Los estudios fenológicos en plantas vasculares: (i) registran y analizan fases fenológicas bien establecidas, tales como períodos de floración, fructificación, dispersión y germinación de semillas; (ii) presentan una extensa historia de registros y estudios botánicos aplicados en agricultura, fruticultura y silvicultura (Chmielewski et al. 2004, Sparks et al. 2005) y (iii) en Chile incluyen detallados estudios ecológicos en ecosistemas altoandinos (Arroyo et al. 1981, Rozzi et al. 1997) y en los bosques templados (Rivero 1991, Smith-Ramírez et al. 1994, Anderson et al. 2006). En contraste, los estudios fenológicos en plantas no vasculares (i) son escasos (Stark 1985, 2002, Glime 2007), (ii) las fases fenológicas definidas varían ampliamente entre autores y según las preguntas de investigación (Greene 1960, Forman 1965, Longton 1979, Imura 1994, Stark 2002) y (iii) en Chile se constata una carencia de estudios fenológicos detallados para musgos (Jofre 2009).
En este artículo presentamos un primer estudio fenológico para una especie de musgo endémica de los bosques templados del sur de Sudamérica (sensu Armesto et al. 1996, 1998), Tayloria dubyi Broth, 1903 (Splachnaceae).

Los estudios fenológicos realizados en diversas localidades y años, requieren un modo consistente de registrar y describir las fases del ciclo anual de los musgos. Sin embargo, esta labor se dificulta debido a que en la mayoría de los estudios sobre flora de briofitas de otras regiones (e.g., Japón, Escandinavia, Norteamérica y Sudamérica) no se mencionan datos fenológicos referente a los períodos de producción y dispersión de esporas y otros eventos del ciclo de vida, relevantes para el conocimiento de estas plantas (Glime 2007).

En este primer estudio fenológico para una especie de musgo, definimos cinco eventos (fenofases) fácilmente observables con lupa de mano en la fase esporofítica de T. dubyi. Esta investigación se realizó durante los años 2007 y 2008, en dos sitios de estudio ubicados en la Reserva de Biosfera Cabo de Hornos (RBCH).

\section{MÉTODOS}

\section{Especie en estudio}

El género Tayloria comprende alrededor de 40 especies distribuidas en zonas de alta montaña, boreales y templadas del mundo (Crosby et al. 1999, Goffinet 2006). En el bioma de los bosques templados de Sudamérica han sido identificadas cuatro especies de Tayloria: T. dubyi, T. stenophysata (Herzog), T. mirabilis (Cardot) y $T$. magellanica (Brid.) (Koponen 1982). Esta última especie se extiende desde Cabo de Hornos a lo largo de los ecosistemas altoandinos hasta el páramo ecuatoriano. En cambio, T. mirabilis, T. stenophysata y $T$. 
dubyi son endémicas para el bioma de los bosques templados de Sudamérica. Más aún, T. dubyi es endémica a las formaciones de turberas de Sphagnum en la ecorregión subantártica de Magallanes en el extremo suroeste de Chile y Argentina (sensu Mittermeier et al. 2004, Rozzi et al. 2006). Las localidades en que esta especie ha sido detectada y colectada incluyen la zona de los canales de Aysén, islas de la $\mathrm{RBCH}$ y el margen oeste de Isla Grande de Tierra del Fuego (Matteri 1985, Rozzi et al. 2006, Goffinet et al. 2006, Jofre 2009) (Fig. 1). En la isla Navarino, T. dubyi es una especie escasa que ha sido detectada sobre fecas de ganso silvestre Chloephaga picta Gmeil, 1789 (Aves, Anatidae) (Caiquén) distribuida sobre y solo en hábitats de turbera en el sector sur aledaño a la bahía Windhond, y en parches de turbera aledaños a la costa norte en la zona de transición subantártica-trasandina de la RBCH (Buck 2002, Rozzi et al. 2006, Jofre 2009) (Fig. 1).

Tayloria dubyi se caracteriza por su gametofito con hojas pequeñas y obtusas y su esporofito de aproximadamente $15 \mathrm{~mm}$ de longitud, posee una hipófisis oscura, cápsulas rojas brillantes y esporas de carácter pegajoso de color anaranjado en su madurez. Los individuos de $T$. dubyi forman pequeños cojines compactos, cuyo diámetro varía de 1.5 a $5 \mathrm{~cm}$ de largo y de 2 a $4.3 \mathrm{~cm}$ de ancho en los sitios estudiados de la Reserva de Biosfera Cabo de Hornos (Jofre 2009).

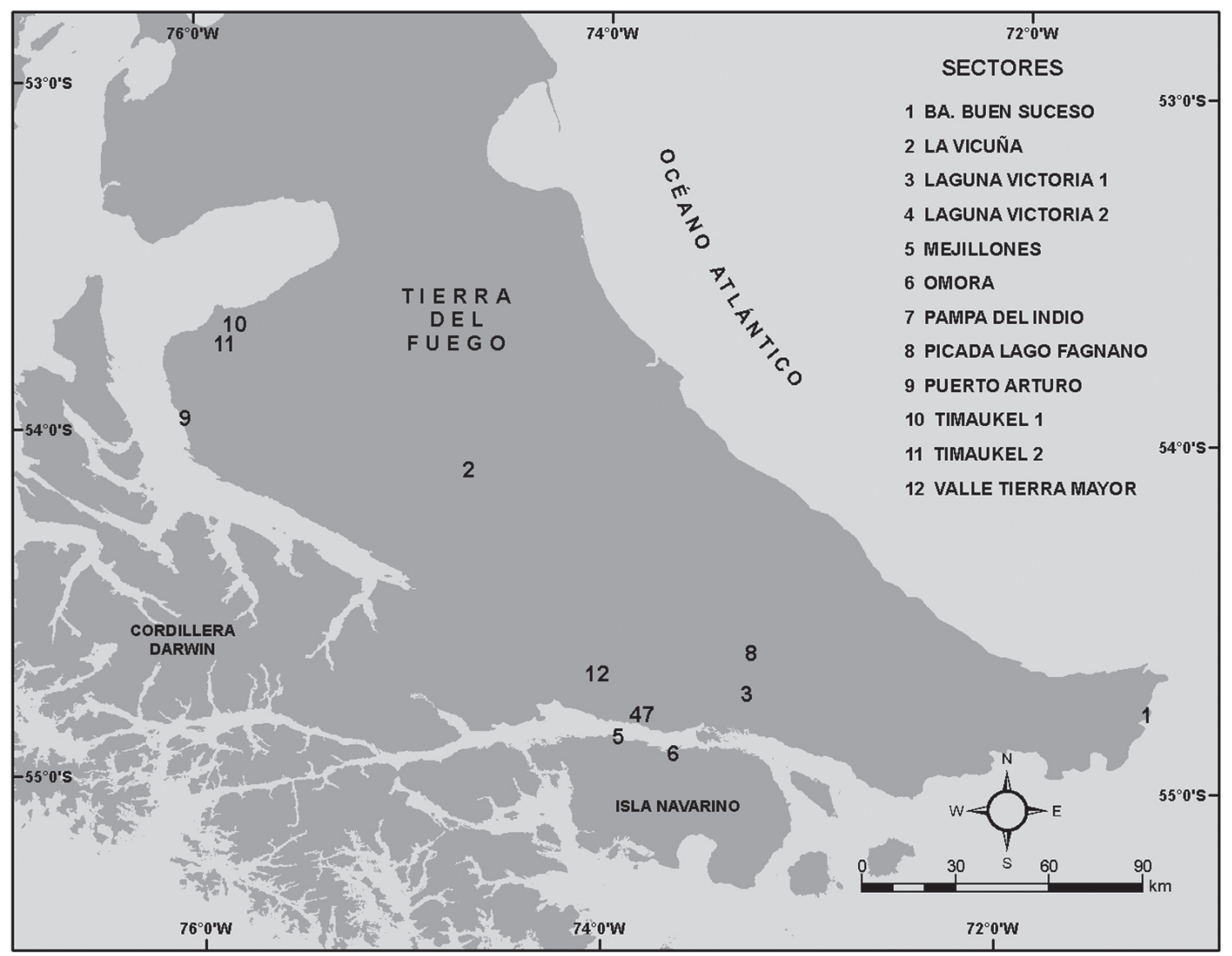

Fig. 1: Mapa mostrando los sitios de estudio en la Isla Navarino, Sitio Mejillones y Sitio Parque Omora (números 5 y 6, respectivamente), y los sitios en la Isla Grande de Tierra del Fuego donde Tayloria dubyi fue localizada por Matteri (1985).

Map showing the study sites in Navarino Island: Parque Omora and Mejillones (numbers 5 and 6, respectively) and in Isla Grande de Tierra del Fuego where Tayloria dubyi was collected by Matteri (1985). 


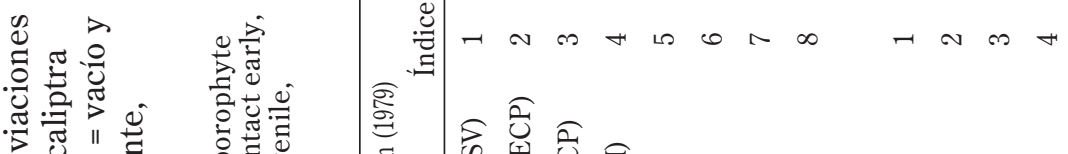

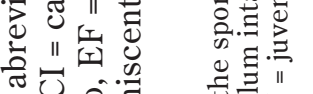

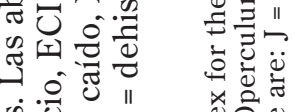

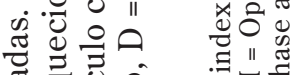

चु.

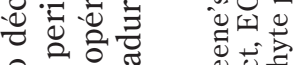

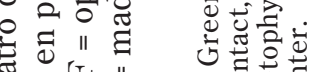

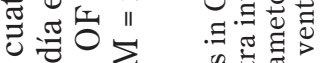

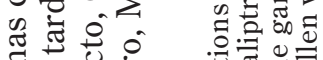

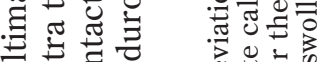

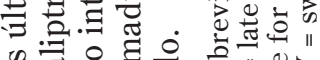

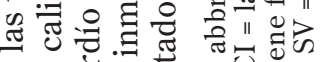

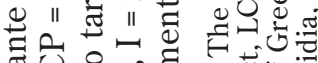

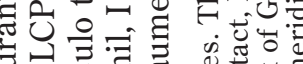

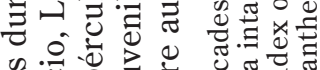

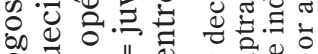

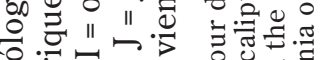
농 等, 品

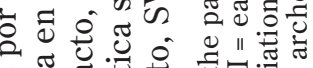

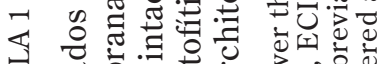

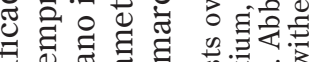
E

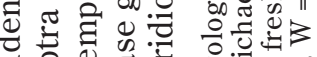

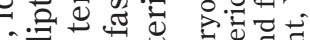

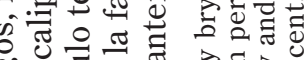
of in

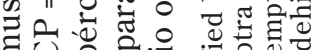

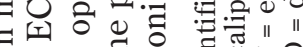
5留

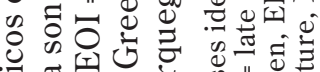

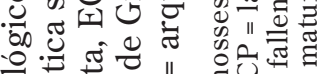

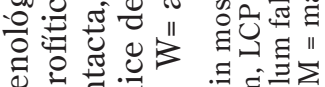

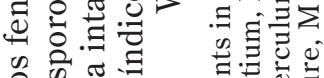

政

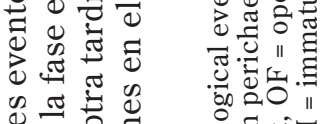

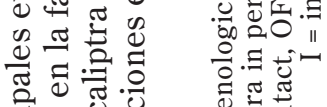

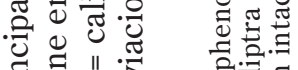

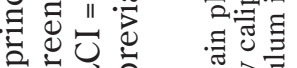

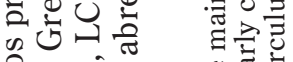

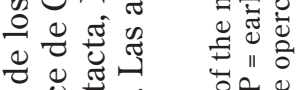

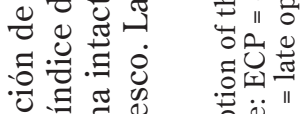

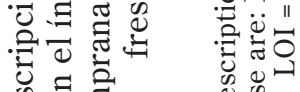

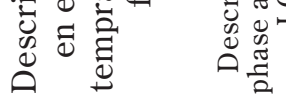

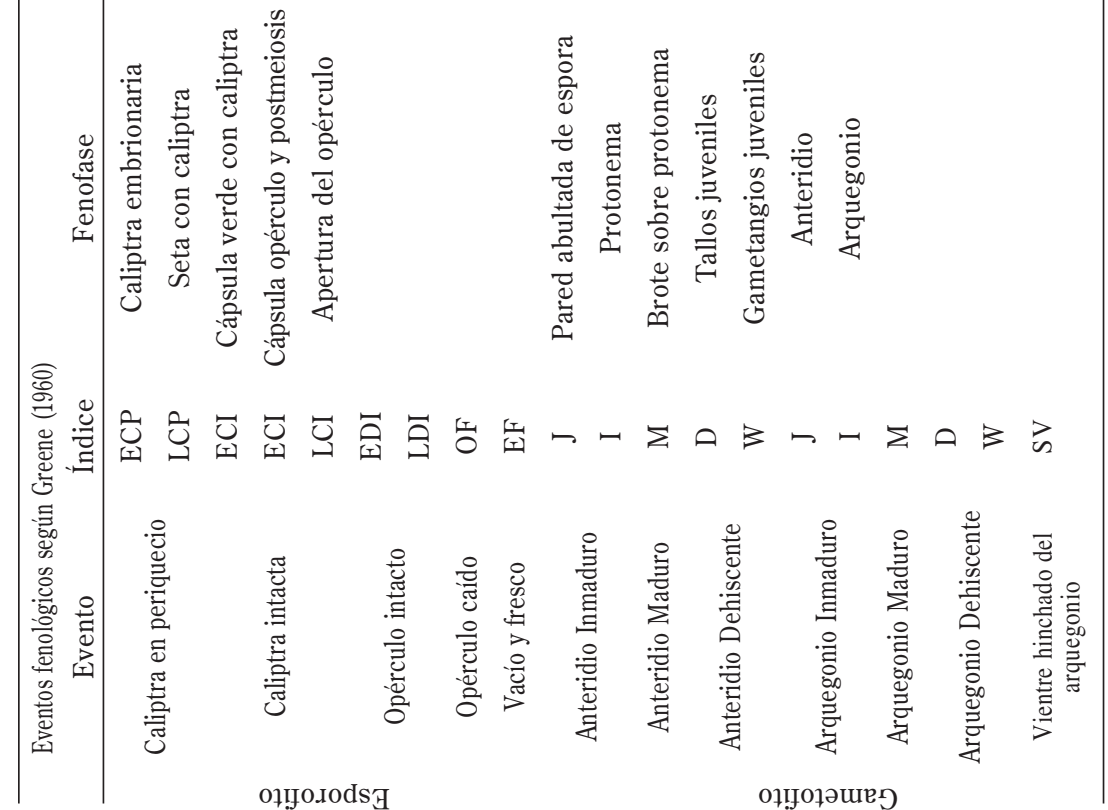




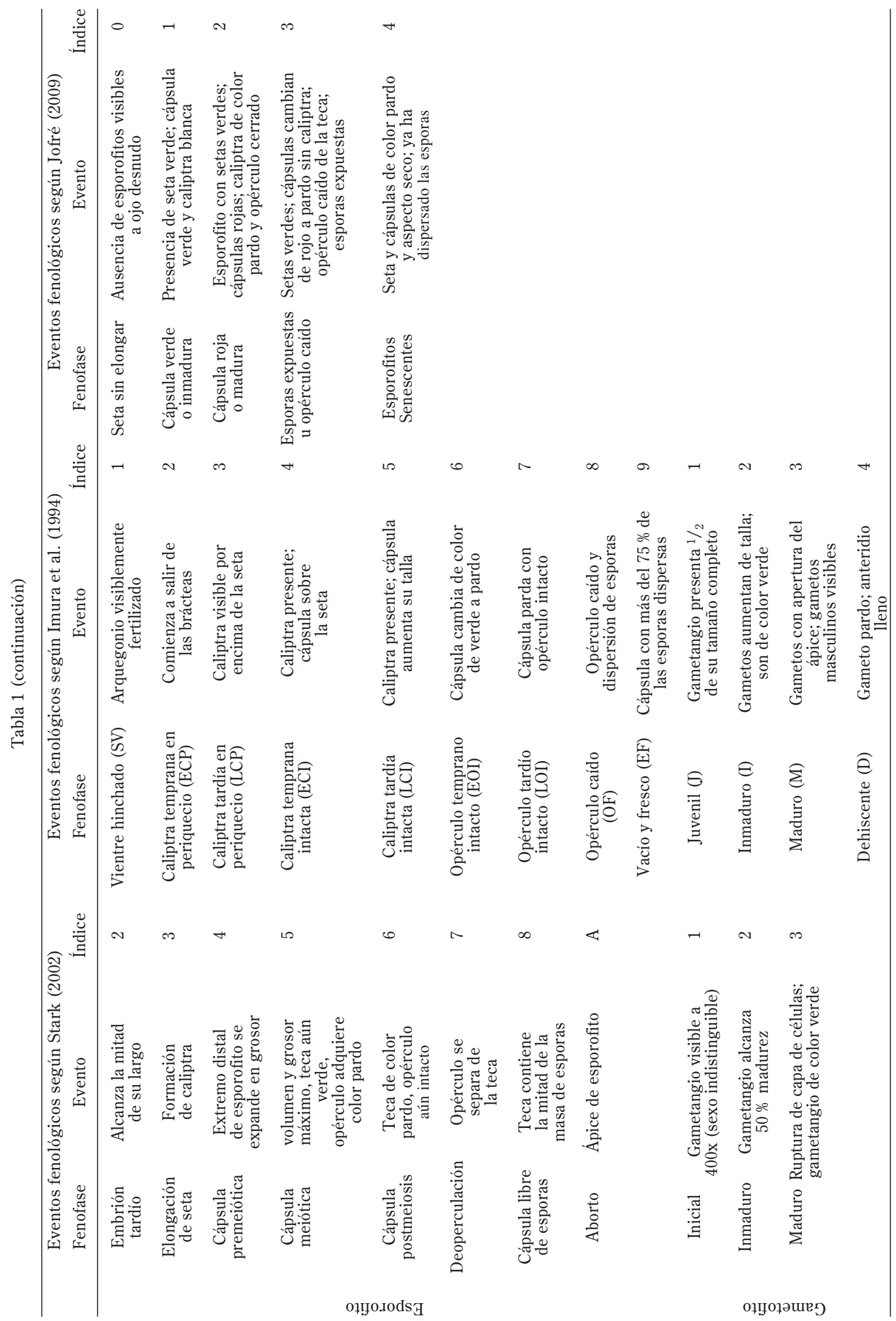




\section{Área de estudio}

Los sitios de estudio se ubican en la costa norte de isla Navarino $\left(55^{\circ} \mathrm{S}\right)$, en la $\mathrm{RBCH}$ en la Región de Magallanes y Antártica Chilena (Fig. 1). Esta zona posee un tipo de clima trasandino con degeneración esteparia y se ubica al oriente de la gran zona de tundra isotérmico que es extremadamente lluviosa (Pisano 1983). La temperatura media anual es de $6{ }^{\circ} \mathrm{C}$ y una precipitación anual de $467 \mathrm{~mm}$ (datos registrados en la ciudad de Puerto Williams, ubicada en la costa norte de la isla Navarino). En esta área existen formaciones de turbera de Sphagnum que se encuentran inmersas en un mosaico de bosques siempreverdes dominados por coigüe de Magallanes (Nothofagus betuloides) (Mirb.) Oerst y por bosques mixtos de coigüe y la especie decidua lenga $(N$. pumilio) (Poepp. et Endl.) (Rozzi et al. 2004).

En la costa norte de isla Navarino se realizó una búsqueda intensiva de poblaciones de $T$. dubyi, detectándose solo en dos sitios. El primero, denominado "sitio Mejillones" (54 53 '56” S; $67^{\circ} 57^{\prime} 55^{\prime \prime}$ W) se ubica a $25 \mathrm{~km}$ al oeste de Puerto Williams y a $1 \mathrm{~km}$ al este de la bahía Mejillones. Este sitio se encuentra a 40 metros sobre el nivel del mar y corresponde a una turbera de Sphagnum magellanicum (Brid.) y $S$. fimbriatum (Wilson) con un área de $80 \mathrm{~m}$ de largo por $19 \mathrm{~m}$ de ancho. El segundo, denominado "sitio Parque Omora" (54 $56^{\circ} 37^{\prime}$ " S; $\left.67^{\circ} 38^{\prime} 49^{\prime \prime} \mathrm{W}\right)$, está ubicado a $3 \mathrm{~km}$ al oeste de la ciudad de Puerto Williams, a un costado de la laguna interior del Parque Etnobótanico Omora que forma parte de la Red Chilena de Sitios de Estudios Socio-Ecológicos a Largo Plazo (Anderson et al. 2008). El sitio se encuentra a 27 metros sobre el nivel del mar y corresponde a una turbera de Sphagnum magellanicum con un área de $50 \mathrm{~m}$ de largo por $22 \mathrm{~m}$ de ancho.

En ambos sitios se realizó un censo del total de cojines de $T$. dubyi, detectándose 22 cojines en el sitio Mejillones y 12 en el sitio Parque Omora. Del total de 44 cojines detectados, en este estudio se utilizaron 18 cojines de T. dubyi: 12 en el sitio Mejillones y 6 en el sitio Parque Omora.

\section{Estudio de fenología}

Para estudiar la secuencia fenológica asociada a la producción y dispersión de esporas de $T$. dubyi, se definieron cinco fases en el desarrollo del esporofito, todas fácilmente observables con lupa de mano (con aumento 10x) en terreno. Estas fenofases se distinguen por las siguientes características:

Fenofase 0 (seta sin elongar): se distingue por la ausencia de esporofitos visibles a ojo desnudo. Bajo lupa estereoscópica (40x) es posible observar el esporofito como una pequeña protuberancia oscura con una caliptra en estado temprano de desarrollo, emergiendo desde el arquegonio. Esta fenofase es equivalente a "embrión tardío" definido por Stark (2002), a "caliptra embriónica" definido por Forman (1965), y a "caliptra temprana en el periquecio del arquegonio" definida por Greene (1960), Longton (1979) e Imura et al. (1994) (véase Tabla 1).

Fenofase 1 (cápsulas verdes o inmaduras): se distingue por la presencia de setas elongadas con cápsulas verdes y caliptra blanca. Esta fenofase es equivalente a la fase de "cápsula premeiótica" (Stark 2002), a “cápsula verde con caliptra” (Forman 1965) y “caliptra tardía intacta” (Greene 1960, Longton 1979, Imura et al. 1994) (Tabla 1).

Fenofase 2 (cápsulas rojas o maduras): se distingue por presentar esporofitos con setas verdes, cápsulas rojas o maduras y caliptra de color pardo, con el opérculo cerrado. Esta fenofase es equivalente a la fase de "cápsula postmeiótica” (Stark 2002), “cápsula operculada y postmeiótica” (Forman 1965), y “opérculo intacto” (Longton 1979) u "opérculo tardío intacto" (Greene 1960, Imura et al. 1994) (Tabla 1).

Fenofase 3 (esporas expuestas u opérculo caído): se distingue por presentar esporofitos con setas verdes a pardo, cápsulas rojas desprovistas de caliptra, en las que el opérculo se ha desprendido de la teca y las esporas han quedado expuestas. Esta fenofase es equivalente a la fase de “deoperculación” (Stark 2002), y “opérculo caído” (Longton 1979, Greene 1960, Imura et al. 1994), y está comprendida dentro de la fase "cápsula deoperculada" (Forman 1965). A diferencia de nuestra "Fenofase 3 ", que en concordancia con Stark (2002) se caracteriza por contener esporas expuestas, Forman (1965) incluye en su fase "cápsula deoperculada” también a cápsulas que carecen de esporas (Tabla 1). 
Fenofase 4 (esporofito senescente): se distingue por presentar esporofitos de color pardo, de aspecto seco y que ya han dispersado sus esporas. Esta fenofase es equivalente a la fase de "cápsula vacía" (Stark 2002), fase de "cápsulas vacías y frescas" (Greene 1960, Longton 1979, Imura et al. 1994) y "espora de pared abultada" (Forman 1965) (Tabla 1).

La presencia o ausencia de esporofitos en los cojines de $T$. dubyi fue evaluada mensualmente (cinco días por cada mes) desde octubre 2007 a abril de 2008 en ambos sitios de estudio. El período en el que no se observaron esporofitos se definió como fenofase 0 , mientras que cuando se observaron esporofitos a ojo desnudo, se procedió a colocar un cuadrante de 5 x $5 \mathrm{~cm}$ y se contó el número total de esporofitos con cápsulas inmaduras (verdes; fenofase 1) y maduras (rojas; fenofase 2) dentro del cuadrante. Luego, las cápsulas maduras fueron examinadas con lupa 10x para distinguir aquellas con opérculo caído y esporas expuestas (fenofase 3 ).

\section{RESULTADOS}

La fase esporofítica de Tayloria dubyi se registró solo entre los meses de noviembre y marzo en la Reserva de Biosfera Cabo de Hornos. El registro de las cinco fenofases definidas más arriba permitió determinar la siguiente secuencia mensual de eventos fenológicos a lo largo de un período anual.

Fenofase 0: seta sin elongar (Fig. 2A). Desde mediados de otoño (abril) hasta mediados de primavera (octubre), en ambos sitios de estudio, solo se observaron cojines de T. dubyi sin esporofitos visibles a ojo desnudo. Bajo lupa estereoscópica (40x) se observaron caliptras en estado embrionario (sensu Forman 1965), de manera que los esporofitos se presentaron como una pequeña protuberancia.

Fenofase 1: cápsulas verdes o inmaduras (Fig. 2B y 2C). Los cojines de T. dubyi presentaron setas elongadas con cápsulas verdes y caliptra blanca en noviembre. Durante este mes, el $100 \%$ de los esporofitos registrados se encontraba en esta fenofase. Esporofitos inmaduros también fueron registrados en diciembre en el sitio Mejillones (33\% del total de esporofitos contabilizados en ese mes) (Fig. 3). Sin embargo, en el sitio Parque Omora solo se observaron esporofitos inmaduros en noviembre (Fig. 4).

Fenofase 2: cápsulas rojas o maduras (Fig. 2D). La presencia de esporofitos con cápsulas de color rojo intenso se detectó principalmente durante los meses de diciembre y enero en ambos sitios de estudio. Los esporofitos maduros representaron más del $60 \%$ de los esporofitos totales contabilizados en diciembre y enero en el sitio Mejillones (Fig. 3), mientras que en el sitio Parque Omora los esporofitos maduros representaron un $57 \%$ y un $33 \%$ de los esporofitos totales respectivamente (Fig. 4). Durante estos dos meses, en ambos sitios de estudio, los esporofitos maduros coexistieron con esporofitos con opérculos caídos y esporas expuestas (fenofase 3) (Fig. 3 y Fig. 4).

Fenofase 3: esporas expuestas u opérculo caído (Fig. 2E). La presencia de esporofitos con cápsulas rojas desprovistas de opérculo comenzó en diciembre y se extendió hasta febrero, alcanzando un máximo en enero en ambos sitios de estudio (Fig. 3 y Fig. 4).

Fenofase 4: esporofito senescente (Fig. 2F). La aparición de esporofitos senescentes (secos y sin esporas) comenzó en febrero extendiéndose hasta el mes de abril en ambos sitios de estudio, observándose un $100 \%$ de esporofitos senescentes en marzo y abril (Fig. 3 y Fig. 4).

\section{DISCUSIÓN}

Los estudios fenológicos en plantas no vasculares que investigan los ciclos reproductivos a lo largo del año y su posible relación con parámetros ambientales (Lloret 1987) son escasos en Chile (Jofre 2009). Nuestro estudio aporta, por un lado, información básica sobre el ciclo de vida de una especie perteneciente a la abundante y diversa flora no vascular del extremo austral de Chile. Por otro, contribuye al desarrollo de futuros estudios fenológicos en plantas no vasculares al definir claramente cinco fenofases de la fase esporofítica que son fácilmente distinguibles con lupa de mano en terreno, y podrían por tanto ser útiles para estudiar otras especies de musgos en Chile. 

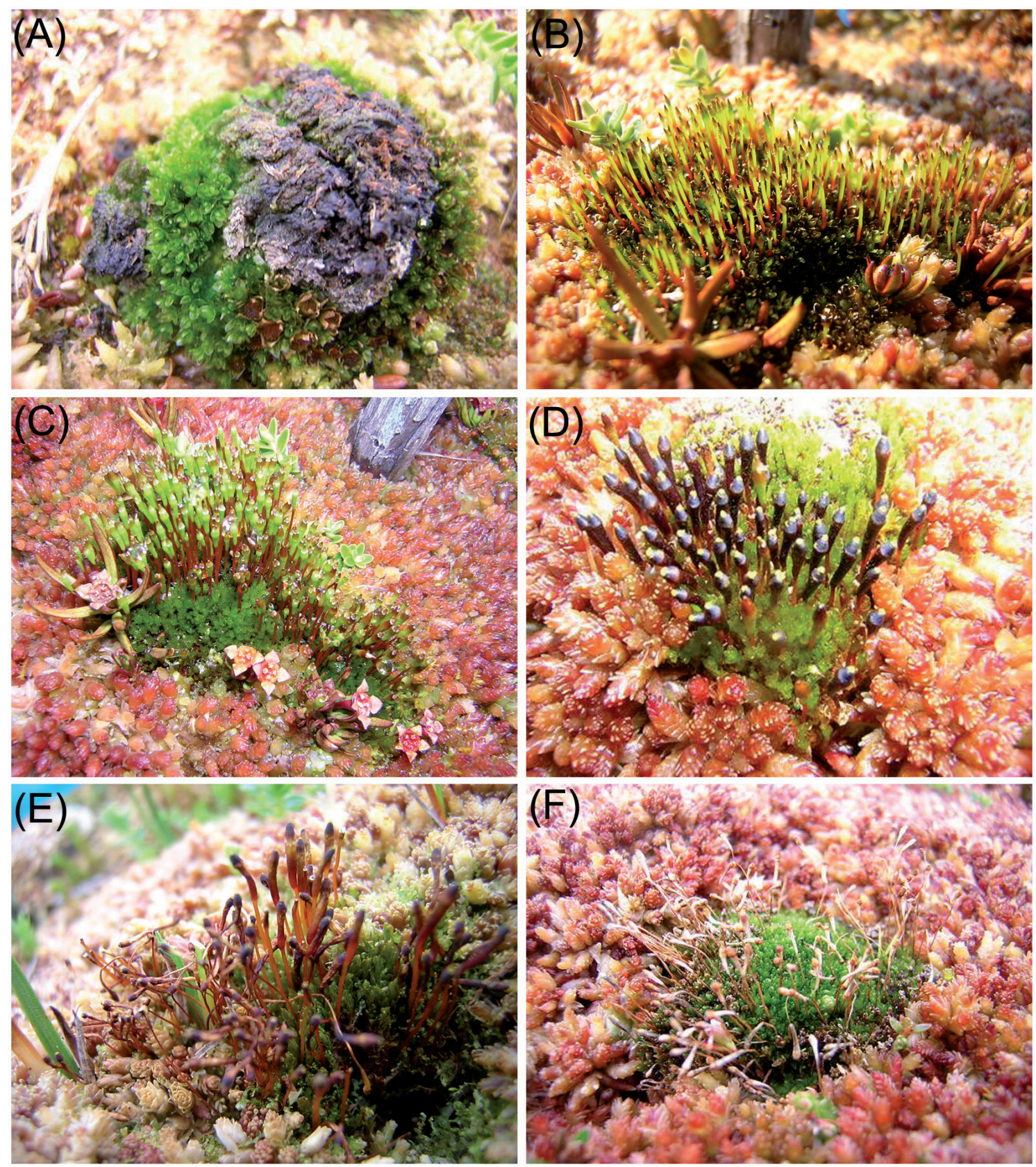

Fig. 2: Cinco fenofases de Tayloria dubyi desde la fenofase 0 (octubre 2007) a la fenofase 4 (abril 2008). (A) fenofase 0 , ausencia de esporofitos visibles a ojo desnudo. (B, C) fenofase 1, esporofitos con cápsulas inmaduras de color verde claro y caliptra blanca, (D) fenofase 2, esporofitos con cápsulas maduras (E) fenofase 3, cápsulas desprovistas de opérculo con exposición de esporas. (F) fenofase 4, esporofitos senescentes.

Five phenophases of Tayloria dubyi from phenophase 0 (octubre 2007) to phenophase 4 (april 2008). (A) phenophase 0 , no visible sporophyte at naked eye. $(B, C)$ phenophase 1 , sporophyte with immature light green capsules and white caliptra; (D) phenophase 2, sporophyte with mature capsules, (E) phenophase 3, capsule without operculum, spore mass exposed. (F) phenophase 4, senescent sporophyte. 
Fenofase $0 \square$ Fenofase 1 घenofase $2 \square$ Fenofase 3 घenofase 4

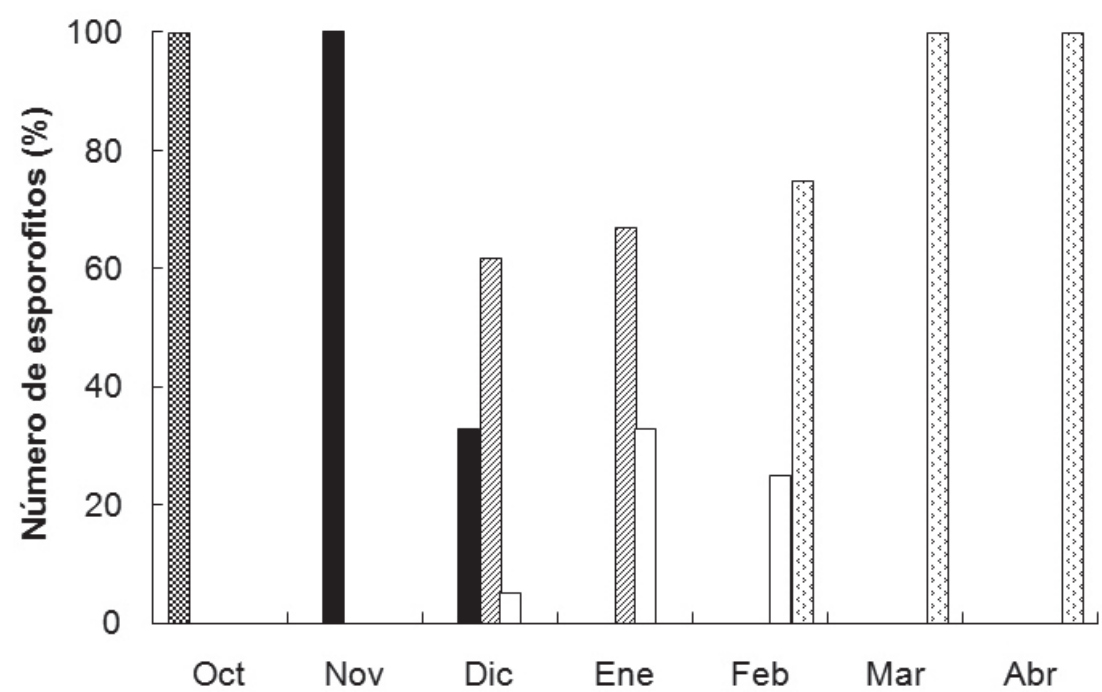

Fig. 3: (A) Secuencia anual de eventos fenológicos en la fase esporofítica de Tayloria dubyi, en el sitio Mejillones ubicado en la costa norte de Isla Navarino, Reserva de Biosfera Cabo de Hornos, Chile, entre octubre 2007 y abril 2008. (B) Secuencia anual de eventos fenológicos en la fase esporofítica de Tayloria dubyi, en el sitio Parque Omora ubicado en la costa norte de isla Navarino, Chile, Reserva de Biosfera Cabo de Hornos, entre octubre 2007 y abril 2008.

(A) Annual sequence of phenological events in the sporophytic phase of Tayloria dubyi, in Mejillones site on the northern coast of Navarino Island, Cape Horn Biosphere Reserve, between October 2007 and April 2008. (B) Annual sequence of phenological events in the sporophitic phase of Tayloria dubyi at Omora Park site, on the northern coast of Navarino Island, Cape Horn Biosphere Reserve, between October 2007 and April 2008.

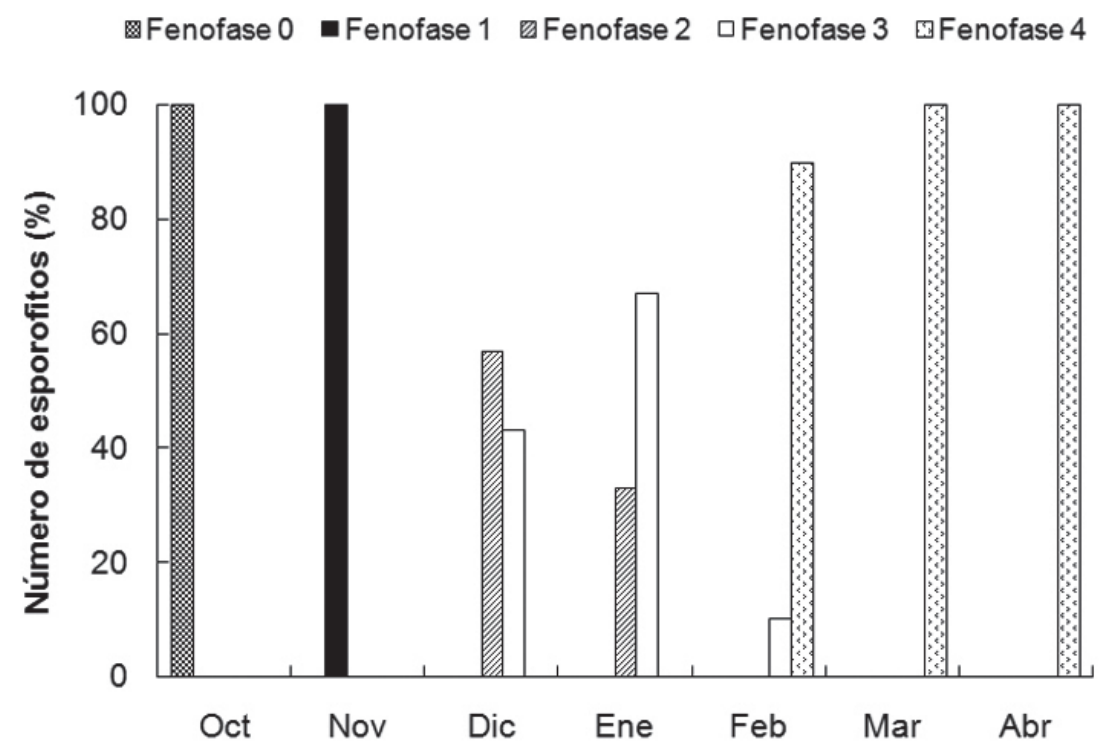

Fig. 4: Secuencia anual de eventos fenológicos en la fase esporofítica de Tayloria dubyi en el sitio Parque Omora ubicado en la costa norte de isla Navarino, Chile.

Annual sequence of phenological events in Tayloria dubyi sporophitic phase in Parque Omora site located on the north coast of Isla Navarino, Chile. 
Las poblaciones de $T$. dubyi estudiadas en la $\mathrm{RBCH}$ presentan un marcado patrón estacional en su fase esporofítica, con una duración total de aproximadamente cinco meses (noviembremarzo). Durante este período los esporofitos crecen, maduran, liberan sus esporas y finalmente mueren. En otras regiones del mundo con estacionalidad menos marcada, la fase esporofítica de algunas especies de musgo se extiende por períodos muy prolongados. Por ejemplo, en los montes Pirineos de España se ha registrado que en algunas poblaciones de Orthotrichum rupestre (Schleich.) la fase esporofítica se extiende por un período de aproximadamente nueve meses (Lloret 1987).

T. dubyi detendría su fase esporofítica en la temporada invernal, probablemente debido a la disminución de la temperatura y a la reducción de la luz solar provocados por la escarcha y nieve que cubren las poblaciones de $T$. dubyi durante el invierno. La detención del desarrollo reproductivo en períodos invernales también sería observada en poblaciones de Pohlia nutans (Hedw.) y P. cruda (Hedw.) de Groenlandia, Georgia del Sur y Gran Bretaña (Greene 1960). En general, este patrón podría observarse en poblaciones de musgos que se encuentran en lugares extremos como zonas subárticas y subantárticas (Longton 1979), así como también en zonas alpinas (Imura 1994).

En las poblaciones de T. dubyi en la isla Navarino, entre mediados de otoño (abril) y mediados de primavera (octubre) no se distinguen esporofitos a ojo desnudo (fenofase 0 : seta sin elongar). Sin embargo, bajo lupa estereoscópica se observaron caliptras en estado embrionario emergiendo desde los arquegonios. Esto indicaría que la reproducción sexual y el desarrollo del embrión de $T$. dubyi se inicia en otoño, y que estos últimos quedarían inactivos durante el invierno como ha sido reportado para musgos de regiones subárticas (Dicranum majus [Turner]) y subantárticas (Polytrichum alpestre [Hoppe]) (Sagmo et al. 1998 y Longton 1972, 1979, respectivamente), así como para Polytrichum ohioense (Renauld \& Cardot) en zonas montañosas de Japón (Ayukawa et al. 2002).

El crecimiento del esporofito de $T$. dubyi sería reanudado a mediados de primavera coincidiendo con el aumento de la luz, fotoperíodo y temperatura, dando paso a la fenofase 1. En su estudio para Tayloria tenuis
(With.) en los pirineos españoles, Lloret (1990) menciona que en primavera, después de romper la pared del arquegonio, el esporofitos comienza a surgir alcanzando su altura máxima en el comienzo del verano para luego iniciar el proceso de maduración. Al iniciarse la fenofase 2, las cápsulas de los esporofito de T. dubyi toman un color rojizo, mientras que el pedicelo y la seta permanecerían de color verde. En este estado, la seta junto con la hipófisis, constituirían un órgano fotosintéticamente eficaz que promovería la rápida maduración de las esporas, como ha sido reportado para las especies Splachnum ampullaceum (Hedw.), S. rubrum (Hewd.) y S. luteum (Hewd.) (Koponen 1990).

La exposición de las esporas o fenofase 3, ocurre a mediados de verano, cuando se alcanzan las máximas temperaturas. Observaciones similares son reportadas para la especie Polytrichum ohioense en la zona altas de Honshu-Japón (Ayukawa 2002), y en algunas especies estudiadas en localidades de los pirineos españoles (Lloret 1987). En general la estación seca sería la más propicia para la dispersión de esporas. Por el contrario, en especies de los géneros Tortula, Syntrichia y Grimmia presentes en el desierto de Arizona, Estados Unidos, este patrón es inverso registrándose dispersión de esporas durante el invierno (Longton 1990).

La fase esporofítica del musgo T. dubyi finaliza con la senescencia de los esporofitos (fenofase 4), lo que fue posible observar a partir de febrero hasta abril en ambos sitios de estudio.

Splachnaceae es la única familia de musgos en que se ha detectado entomocoría, esto es, dispersión de las esporas por insectos, registrándose específicamente dípteros (Koponen 1982, 1990, Lloret 1990, Marino 1991, 1997, Goffinet 2006, Goffinet et al. 2006). Sin embargo, este mecanismo de dispersión biótica de esporas en musgos ha sido estudiado en forma sistemática exclusivamente en el Hemisferio Norte. Datos preliminares sugieren que este fenómeno podría ocurrir en la especie Tayloria purpurascens de Nueva Zelanda (Koponen 1990). La concentración del período de liberación de esporas desde los esporofitos de T. dubyi a comienzos del verano austral en 
coincidencia con los meses de mayor actividad de dípteros potencialmente dispersantes de esporas, sugiere que la entomocoría también podría ocurrir en la Reserva de Biosfera Cabo de Hornos, en la ecorregión subantártica de Magallanes (Jofre 2009). Por lo tanto, nuestro trabajo no solo define una metodología para estudios fenológicos en briofitas australes, sino que también propone evaluar en trabajos futuros la ocurrencia de entomocoría en especies de Splachnaceae que habitan en ecosistemas forestales y turberas en estas latitudes altas del Hemisferio Sur.

\section{AGRADECIMIENTOS}

Los autores agradecen a Juan Carlos Villareal (University of Connecticut), Guilherme Pereira Filho (Universidad de São Paulo) Carlos Olave (CEQUA) sus sugerencias para el análisis de datos y presentación de los mismos; a Juan Armesto (CASEB-P. Universidad Católica de Chile) sus comentarios al manuscrito y la tesis de Magíster de J. Jofre, a Rina Charlin, José Eduardo Morales y el equipo del Parque Etnobotánico Omora su ayuda en el trabajo de terreno. Esta investigación ha sido apoyada por los proyectos PFB-23-(CONICYT) y P05002 (ICM-MIDEPLAN) del Instituto de Ecología y Biodiversidad (IEB) y el Programa de Estudios Socio-Ecológicos a Largo Plazo del Parque Omora (Universidad de Magallanes IEB), y J. Jofre agradece la Beca de Magíster del IEB (Proyecto ICM P05-002).

\section{LITERATURA CITADA}

ANDERSON CB, GE LIKENS, R ROZZI, JR GUTIÉRREZ, JJ ARMESTO \& A POOLE (2008) Integrating Science and Society through LongTerm Socio-Ecological Research. Environmental Ethics 30: 295-312.

AYUKAWA E, S IMURA, S KUDOH \& H KANDA (2002) Reproductive phenology of subalpine moss, Polytrichum ohioense Ren. Et Card. Polar Bioscience 15: 88-96.

ARMESTO JJ, R ROZZI, C SMITH-RAMÍREZ \& MTK ARROYO (1998) Conservation targets in South American temperate forests. Science 282: 12711272.

ARMESTO JJ, MTK ARROYO \& C VILLAGRÁN (1996) Ecología de los bosques nativos de Chile. Editorial Universitaria, Santiago, Chile.

ARROYO MTK, JJ ARMESTO \& C VILLAGRÁN (1981) Plant phenological patterns in the high Andean
Cordillera of Central Chile. Journal of Ecology 69: 205-233.

BUCK WR (2002) Preliminary key to the mosses of Isla Navarino, Chile (Prov. Antártica Chilena). Press New York, New York, USA.

CHMIELEWSKI F, A MÜLLER \& E BRUNS (2004) Agricultural and Forest Meteorology 121: 69-78.

CROSBY MR, RE MAGILL, B ALLEN \& S HE (1999) A checklist of mosses. Missouri Botanical Garden, St. Louis, USA.

FORMAN RTT (1965) A system for studying moss phenology. Bryologist 68: 289-300.

GREENE SW (1960) The maduration cycle or the stages of development of gametangia and capsules in mosses. Transactions of the British Bryological Society 3: 736-745.

GOFFINET B, W BUCK, F MASSARDO \& R ROZZI (2006) Miniature forests of Cape Horn. Publicaciones del Gobierno Regional y Antártica Chilena, Punta Arenas, Chile.

GOFFINET B (2006) Splachnaceae. FL. Australia 51: $173-181$.

GLIME J (2007) Bryophyte ecology. Vol 1. Physiological ecology. Ebook sponsored by Michigan Technological University and the International Association of Bryologists, Michigan.

IMURA S (1994) Phenological study in two dioecious mosses, Atrichum rhystophyllum (C. Mull.) Par. and Pogonatum inflexum (Lindb.) Lac. Journal Hattori Botanical Laboratory 76: 105-114.

JOFRE J (2009) Fenología del musgo Tayloria dubyi en las turberas de la Reserva de Biosfera Cabo de Hornos: ¿Un caso de entomofilia? Tesis de Magíster, Facultad de Ciencias, Universidad de Magallanes, Punta Arenas, Chile.

KOPONEN A (1990) Entomophily in the Splachnaceae. Botanical Journal of the Linnean Society 104 : 115-127.

KOPONEN A (1982) The family Splachnaceae in Australasia and the Pacific. Journal of the Hattori Botanical Laboratory 52: 87-91.

LONGTON RE (1990) Sexual reproduction in bryophytes in relation to physical factors of the environment. En: Chopa RN \& SC Bratla (eds) Bryophyte development: Physiology and biochemistry: 139-166. CRC Press, Boca Raton, FL.

LONGTON RE (1979) Studies on growth, reproduction and population ecology in relation to microclimate in the bipolar moss Polytrichum alpestre. The Bryologist 82: 325-367.

LONGTON RE (1972) Reproduction of Antarctic mosses in the genera Polytrichum and Psilopilum with particular reference to temperature. British Antarctic Survey Bulletin 27: 51-96.

LLORET F (1990) Colonización de excrementos de bóvido por Tayloria tenuis (With.) Schimp. Anales Jardín Botánico de Madrid 46: 469-476.

LLORET F (1987) Efecto de la altitud sobre la fenología de briofitos en el Pirineo Oriental. Anales del Jardín Botánico de Madrid 43: 203215.

MARINO P (1991) Dispersal and coexistence of mosses (Splachnaceae) in patches habitat. Journal of Ecology 79: 1047-1060.

MARINO P (1997) Competition, dispersal and coexistence of Splachnaceae in patchy habitats. Advances in Bryology 6: 241-263.

MARINO P, R RAGUSO \& B GOFFINET (2008) The 
ecology and evolution of fly dispersed dung mosses (Family Splachnaceae): Manipulating insect behaviour through odour and visual cues. Symbiosis 47: 61-76.

MATTERI CM (1985) Catálogo de los musgos. En: Boelcke O, DM Moore \& FA Roig (eds) Transecta botánica de la Patagonia austral: 265298. Buenos Aires, Consejo Nacional de Investigaciones Científicas y Técnicas (Argentina), Instituto de la Patagonia (Chile) and Royal Society (Gran Bretaña).

MITTERMEIER R, P ROBLES, M HOFFMAN, J PILGRIM, T BROOKS, C MITTERMEIER, J LAMOREUX \& G DA FONSECA (2004) Hotspot revisited: Earth's biologically richest and most endangered terrestrial ecoregions. University of Chicago Press, Chicago, IL.

PISANO E (1983) The Magellanic Tundra complex. En: Gore AJP (ed) Mires: Swamp, bog, fen and moor. B. Regional Studies 10: 295-329. Elsevier Sc. Publ. Co. Amsterdam, The Netherlands.

PHARO E \& CE ZARTMAN (2007) Bryophytes in a changing landscape: The hierarchical effects of habitat fragmentation on ecological and evolutionary processes. Biological Conservation 35: 315-325.

RIVEROS M (1991) Biología reproductiva en especies vegetales de dos comunidades de la zona templada del sur de chile, $40^{\circ} \mathrm{S}$. Tesis de Magíster, Facultad de Ciencias, Universidad de Chile, Santiago, Chile.

ROZZI R, JJ ARMESTO, B GOFFINET, W BUCK, F MASSARDO et al. (2008) Changing lenses to assess biodiversity: Patterns of species richness in sub-antarctic plants and implications for global conservation. Frontiers Ecological Environment 6: 131-137.

ROZZI R, F MASSARDO, CB ANDERSON, K HEIDINGER \& J SILANDER JR (2006) Ten principles for biocultural conservation at the southern Tip of the Americas: The approach of the Omora Ethnobotanical Park. Ecology \& Society 11: 43 pp. (en línea) URL: http:// www.ecologyandsociety.org/vol11/iss1/art43/ (accedido Marzo 3, 2009)

ROZZI R, F MASSARDO \& CB ANDERSON (2004) The Cape Horn Biosphere Reserve: A proposal for conservation and tourism to achieve sustainable development at the southern end of the Americas. Ediciones Universidad de Magallanes, Punta Arenas, Chile.

SAGMO S, L SÖDERSTÖRM, S BAKKEN, KI FLATBERG \& B PEDERSEN (1998) Reproductive phenology of Dicranum majus in central Norway. Journal of Bryology 20: 311-321.

SMITH-RAMÍREZ C \& JJ ARMESTO (1994) Flowering and fruiting patterns in the temperate rain forest of Chiloé: Ecologies and climatic constraints. Journal of Ecology 82: 353-365.

SPARKS TH, PJ CROXTON, N COLLINSON \& $\mathrm{P}$ TAYLOR (2005) Example of phenological change, past and present, in UK farming. Annals of Applied Biology 4: 531-537.

STARK LR (2002) Phenology and its repercussions on the reproductive ecology of mosses. The Bryologist 105: 204-218.

STARK LR (1985) Phenology and species concepts: A case study. The Bryologist 88: 190-198.

Editor Asociado: Ernesto Gianoli

Recibido el 30 de abril de 2009; aceptado el 21 de octubre de 2009 